\title{
Degenerate Hermite poly-Bernoulli numbers and polynomials with q-parameter
}

\author{
Waseem A. Khan, Idrees A. Khan and Musharraf Ali
}

\begin{abstract}
In this paper, we introduce a new class of degenerate Hermite polyBernoulli polynomials with q-parameter and give some identities of these polynomials related to the Stirling numbers of the second kind. Some implicit summation formulae and general symmetry identities are derived by using different analytical means and applying generating functions. These results extend some known summations and identities of degenerate Hermite poly-Bernoulli numbers and polynomials.
\end{abstract}

Mathematics Subject Classification (2010): 11B68, 11B73, 11B75, 33C45.

Keywords: Hermite polynomials, degenerate q-poly-Bernoulli polynomials, degenerate Hermite q-poly-Bernoulli polynomials, summation formulae, symmetric identities.

\section{Introduction}

The special polynomials of more than one variable provide new means of analysis for the solution of wide class of partial differential equations often encountered in physical problems. The importance of multi-variable Hermite polynomials has been recognized [6] and these polynomials have been exploited to deal with quantum mechanical and optical beam transport problems.

It happens very often that the solution of a given problem in physics or applied mathematics requires the evaluation of infinite sums, involving special functions. Problems of this type arise, for example, in the computation of the higher-order moments of a distribution or to evaluate transition matrix elements in quantum mechanics. In $[7],[8],[9],[19],[20],[21],[22]$, it has been shown that the summation formulae of special functions, encountered in applications ranging from electromagnetic process to combinatorics can be written in terms of Hermite polynomials of more than one variable. 
The 2-variable Kampe de Feriet generalization of the Hermite polynomials [3] and [8] are defined as

$$
H_{n}(x, y)=n ! \sum_{r=0}^{\left[\frac{n}{2}\right]} \frac{y^{r} x^{n-2 r}}{r !(n-2 r) !} .
$$

These polynomials are specified by the generating function:

$$
e^{x t+y t^{2}}=\sum_{n=0}^{\infty} H_{n}(x, y) \frac{t^{n}}{n !}
$$

and reduce to the ordinary Hermite polynomials $H_{n}(x)$ (see [1]) when $y=-1$ and $x$ is replaced by $2 x$.

In (2016), Khan [13] introduced the degenerate Hermite polynomials of two variables by means of the following generating function:

$$
(1+\lambda t)^{\frac{x}{\lambda}}\left(1+\lambda t^{2}\right)^{\frac{y}{\lambda}}=\sum_{n=0}^{\infty} H_{n}(x, y ; \lambda) \frac{t^{n}}{n !},
$$

where $\lambda \neq 0$. Since $(1+\lambda t)^{\frac{1}{\lambda}} \longrightarrow e^{t}$ as $\lambda \longrightarrow 0$, it is evident that (1.3) reduces to (1.2). That is $H_{n}(x, y)$ limiting case of $H_{n}(x, y ; \lambda)$ when $\lambda \longrightarrow 0$.

The following representation of degenerate Hermite polynomials $H_{n}(x, y ; \lambda)$ is given by

$$
H_{n}(x, y ; \lambda)=n ! \sum_{r=0}^{\left[\frac{n}{2}\right]} \frac{\left(-\frac{x}{\lambda}\right)_{n-2 r}\left(-\frac{y}{\lambda}\right)_{r}(-\lambda)^{n-r}}{r !(n-2 r) !} .
$$

Since $\lim _{\lambda \rightarrow 0} H_{n}(x, y ; \lambda)=H_{n}(x, y),(1.1)$ is a limiting case of (1.4).

For $\lambda \in \mathbb{C}$, Carlitz introduced the degenerate Bernoulli polynomials by means of the following generating function:

$$
\frac{t}{(1+\lambda t)^{\frac{1}{\lambda}}-1}(1+\lambda t)^{\frac{x}{\lambda}}=\sum_{n=0}^{\infty} \beta_{n}(x ; \lambda) \frac{t^{n}}{n !}, \quad(\text { see }[4],[18],[17])
$$

so that

$$
\beta_{n}(x ; \lambda)=\sum_{n=0}^{m}\left(\begin{array}{l}
n \\
m
\end{array}\right) \beta_{m}(\lambda)\left(\frac{x}{\lambda}\right)_{n-m} .
$$

When $x=0, \beta_{n}(\lambda)=\beta_{n}(0 ; \lambda)$ are called the degenerate Bernoulli numbers.

From (1.5), we note that

$$
\begin{gathered}
\sum_{n=0}^{\infty} \lim _{\lambda \rightarrow 0} \beta_{n}(x ; \lambda) \frac{t^{n}}{n !}=\lim _{\lambda \longrightarrow 0} \frac{t}{(1+\lambda t)^{\frac{1}{\lambda}}-1}(1+\lambda t)^{\frac{x}{\lambda}} \\
=\frac{t}{e^{t}-1} e^{x t}=\sum_{n=0}^{\infty} B_{n}(x) \frac{t^{n}}{n !},
\end{gathered}
$$

where $B_{n}(x)$ are called the Bernoulli polynomials (see [1]-[25]). 
The classical polylogarithm function $\operatorname{Li}_{k}(z)$ is

$$
\operatorname{Li}_{k}(z)=\sum_{m=1}^{\infty} \frac{z^{m}}{m^{k}},(k \in \mathbb{Z}) \quad(\text { see }[13],[14],[16])
$$

so for $k \leq 1$,

$$
\operatorname{Li}_{k}(z)=-\ln (1-z), \operatorname{Li}_{0}(z)=\frac{z}{1-z}, \operatorname{Li}_{-1}(z)=\frac{z}{(1-z)^{2}}, \ldots
$$

The poly-Bernoulli polynomials are given by

$$
\frac{\operatorname{Li}_{k}\left(1-e^{-t}\right)}{e^{t}-1} e^{x t}=\sum_{n=0}^{\infty} B_{n}^{(k)}(x) \frac{t^{n}}{n !}, \quad \text { (see [2], [12], [13]). }
$$

For $k=1$ in (1.9), we have

$$
\frac{\mathrm{Li}_{1}\left(1-e^{-t}\right)}{e^{t}-1} e^{x t}=\frac{t}{e^{t}-1} e^{x t}=\sum_{n=0}^{\infty} B_{n}(x) \frac{t^{n}}{n !} .
$$

From (1.7) and (1.10), we have

$$
B_{n}^{(1)}(x)=B_{n}(x) .
$$

Very recently, Khan [13] introduced the degenerate Hermite poly-Bernoulli polynomials of two variables ${ }_{H} \beta_{n, q}^{(k)}(x, y ; \lambda)$ by means of the following generating function:

$$
\left(\frac{\operatorname{Li}_{k}\left(1-e^{-t}\right)}{(1+\lambda t)^{\frac{1}{\lambda}}-1}\right)^{\alpha}(1+\lambda t)^{\frac{x}{\lambda}}\left(1+\lambda t^{2}\right)^{\frac{y}{\lambda}}=\sum_{n=0}^{\infty} H_{n}^{(k)}(x, y ; \lambda) \frac{t^{n}}{n !},
$$

so that

$$
{ }_{H} B_{n}^{(k)}(x, y ; \lambda)=\sum_{m=0}^{n}\left(\begin{array}{l}
n \\
m
\end{array}\right) \beta_{n-m}^{(k)}(\lambda) H_{m}(x, y ; \lambda) .
$$

The Stirling number of the first kind is given by

$$
(x)_{n}=x(x-1) \cdots(x-n+1)=\sum_{l=0}^{n} S_{1}(n, l) x^{l},(n \geq 0),
$$

and the Stirling number of the second kind is defined by generating function:

$$
\left(e^{t}-1\right)^{n}=n ! \sum_{l=n}^{\infty} S_{2}(l, n) \frac{t^{l}}{l !} .
$$

A generalized falling factorial sum $\sigma_{k}(n ; \lambda)$ can be defined by the generating function $[25]$

$$
\sum_{k=0}^{\infty} \sigma_{k}(n ; \lambda) \frac{t^{k}}{k !}=\frac{(1+\lambda t)^{\frac{(n+1)}{\lambda}}-1}{(1+\lambda t)^{\frac{1}{\lambda}}-1}
$$

Note that

$$
\lim _{\lambda \rightarrow 0} \sigma_{k}(n ; \lambda)=S_{k}(n)
$$


The object of this paper as follows, we first give definition of the degenerate Hermite poly-Bernoulli polynomials ${ }_{H} \beta_{n}^{(k)}(x, y ; \lambda)$ with q-parameter and then extend and illustrate how, a connection between Hermite and Bernoulli polynomials can yield new expansions and representations. Some implicit summation formulae and general symmetry identities are derived. These results establish a link between these families of polynomials (namely degenerate Hermite and degenerate q-poly-Bernoulli polynomials).

\section{2. q-analogue of degenerate Hermite poly-Bernoulli polynomials}

In this section, we introduce q-analogue of degenerate of Hermite-poly-Bernoulli numbers and polynomials and its properties.

Definition 2.1. For $\lambda \in \mathbb{C}, k \in \mathbb{Z}$ and $n \geq 0,0 \leq q<1$, we introduce q-analogue of degenerate Hermite poly-Bernoulli polynomials by means of the following generating function:

$$
\frac{\operatorname{Li}_{k, q}\left(1-e^{-t}\right)}{(1+\lambda t)^{\frac{1}{\lambda}}-1}(1+\lambda t)^{\frac{x}{\lambda}}\left(1+\lambda t^{2}\right)^{\frac{y}{\lambda}}=\sum_{n=0}^{\infty} H \beta_{n, q}^{(k)}(x, y ; \lambda) \frac{t^{n}}{n !},
$$

where $\operatorname{Li}_{k, q}(t)=\sum_{n=0}^{\infty} \frac{t^{n}}{[n]_{q}^{k !}}$ is the k-th q-polylogarithm function (see [6], [10], [23]).

When $x=y=0$ in $(2.1), \beta_{n}^{(k)}(\lambda)={ }_{H} \beta_{n}^{(k)}(0,0 ; \lambda)$ are called the q-analogue of degenerate poly-Bernoulli numbers.

Note that

and

$$
{ }_{H} \beta_{n, q}^{(1)}(x, y ; \lambda)={ }_{H} \beta_{n, q}(x, y ; \lambda)
$$

$$
\lim _{\lambda \longrightarrow 0} \beta_{n, q}^{(k)}(x, y ; \lambda)={ }_{H} B_{n, q}^{(k)}(x, y) .
$$

Remark 2.2. For $y=0$ in (2.1), the result reduces to the q-analogue of degenerate poly-Bernoulli polynomials of Jung and Ryoo [10, p. 32, Eq. (2.1)] defined as

$$
\frac{\operatorname{Li}_{k, q}\left(1-e^{-t}\right)}{(1+\lambda t)^{\frac{1}{\lambda}}-1}(1+\lambda t)^{\frac{x}{\lambda}}=\sum_{n=0}^{\infty} \beta_{n, q}^{(k)}(x ; \lambda) \frac{t^{n}}{n !},(k \in \mathbb{Z}) .
$$

Theorem 2.3. For $\lambda \in \mathbb{C}, k \in \mathbb{Z}$ and $n \geq 0,0 \leq q<1$, we have

$$
{ }_{H} \beta_{n, q}^{(k)}(x, y ; \lambda)=\sum_{l=0}^{n}\left(\begin{array}{l}
n \\
l
\end{array}\right) \beta_{l, q}^{(k)}(\lambda) H_{n-l}(x, y ; \lambda) .
$$

Proof. By using definition (2.1), we have

$$
\begin{gathered}
\sum_{n=0}^{\infty} H \beta_{n, q}^{(k)}(x, y ; \lambda) \frac{t^{n}}{n !}=\frac{\operatorname{Li}_{k, q}\left(1-e^{-t}\right)}{(1+\lambda t)^{\frac{1}{\lambda}}-1}(1+\lambda t)^{\frac{x}{\lambda}}\left(1+\lambda t^{2}\right)^{\frac{y}{\lambda}} \\
=\sum_{l=0}^{\infty} \beta_{l, q}^{(k)}(\lambda) \frac{t^{l}}{l !} \sum_{n=0}^{\infty} H_{n}(x, y ; \lambda) \frac{t^{n}}{n !}
\end{gathered}
$$




$$
\sum_{n=0}^{\infty}{ }_{H} \beta_{n, q}^{(k)}(x, y ; \lambda) \frac{t^{n}}{n !}=\sum_{n=0}^{\infty}\left(\sum_{l=0}^{n}\left(\begin{array}{c}
n \\
l
\end{array}\right) \beta_{l, q}^{(k)}(\lambda) H_{n}(x, y ; \lambda)\right) \frac{t^{n}}{n !} .
$$

Comparing the coefficients of $\frac{t^{n}}{n !}$ in both sides, we get (2.3).

Theorem 2.4. For $n \geq 0$, we have

$$
{ }_{H} \beta_{n, 1}^{(2)}(x, y ; \lambda)=\sum_{m=0}^{n}\left(\begin{array}{c}
n \\
m
\end{array}\right) \frac{B_{m}}{m+1} H \beta_{n-m}(x, y ; \lambda) .
$$

Proof. Consider equation(2.1), we have

$$
\begin{gathered}
\sum_{n=0}^{\infty} H \beta_{n, 1}^{(k)}(x, y ; \lambda) \frac{t^{n}}{n !}=\frac{\operatorname{Li}_{k, 1}\left(1-e^{-t}\right)}{(1+\lambda t)^{\frac{1}{\lambda}}-1}(1+\lambda t)^{\frac{x}{\lambda}}\left(1+\lambda t^{2}\right)^{\frac{y}{\lambda}} \\
=\frac{(1+\lambda t)^{\frac{x}{\lambda}}\left(1+\lambda t^{2}\right)^{\frac{y}{\lambda}}}{(1+\lambda t)^{\frac{1}{\lambda}}-1} \int_{0}^{t} \underbrace{\frac{1}{e^{z}-1} \int_{0}^{t} \frac{1}{e^{z}-1} \cdots \frac{1}{e^{z}-1} \int_{0}^{t} \frac{z}{e^{z}-1}}_{(k-1)-\text { times }} d z \cdots d z
\end{gathered}
$$

For $k=2$ in $(2.5)$, we have

$$
\begin{gathered}
\sum_{n=0}^{\infty} H_{H, 1}^{(2)}(x, y ; \lambda) \frac{t^{n}}{n !}=\frac{(1+\lambda t)^{\frac{x}{\lambda}}\left(1+\lambda t^{2}\right)^{\frac{y}{\lambda}}}{(1+\lambda t)^{\frac{1}{\lambda}}-1} \int_{0}^{t} \frac{z}{e^{z}-1} d z \\
=\left(\sum_{m=0}^{\infty} \frac{B_{m}}{m+1} \frac{t^{m}}{m !}\right) \frac{(1+\lambda t)^{\frac{x}{\lambda}}\left(1+\lambda t^{2}\right)^{\frac{y}{\lambda}}}{(1+\lambda t)^{\frac{1}{\lambda}}-1} \\
=\left(\sum_{m=0}^{\infty} \frac{B_{m}}{m+1} \frac{t^{m}}{m !}\right)\left(\sum_{n=0}^{\infty} H \beta_{n}(x, y ; \lambda) \frac{t^{n}}{n !}\right) .
\end{gathered}
$$

Replacing $n$ by $n-m$ in above equation, we have

$$
=\sum_{n=0}^{\infty} \sum_{m=0}^{n}\left(\begin{array}{c}
n \\
m
\end{array}\right) \frac{B_{m}}{m+1} H_{n-m}(x, y ; \lambda) \frac{t^{n}}{n !} .
$$

On equating the coefficients of the like powers of $t$ in the above equation, we get the result (2.4).

Theorem 2.5. For $n \geq 0$, we have

$$
{ }_{H} \beta_{n, q}^{(k)}(x, y ; \lambda)=\sum_{p=0}^{n}\left(\begin{array}{c}
n \\
p
\end{array}\right)\left(\sum_{l=1}^{p+1} \frac{(-1)^{l+p+1} l ! S_{2}(p+1, l)}{[l]_{q}^{k}(p+1)}\right){ }_{H} \beta_{n-p}(x, y ; \lambda) .
$$

Proof. From equation (2.1), we have

$$
\sum_{n=0}^{\infty} H \beta_{n, q}^{(k)}(x, y ; \lambda) \frac{t^{n}}{n !}=\left(\frac{\operatorname{Li}_{k, q}\left(1-e^{-t}\right)}{t}\right)\left(\frac{t(1+\lambda t)^{\frac{x}{\lambda}}\left(1+\lambda t^{2}\right)^{\frac{y}{\lambda}}}{(1+\lambda t)^{\frac{1}{\lambda}}-1}\right) .
$$

Now

$$
\frac{1}{t} \operatorname{Li}_{k, q}\left(1-e^{-t}\right)=\frac{1}{t} \sum_{l=1}^{\infty} \frac{\left(1-e^{-t}\right)^{l}}{[l]_{q}^{k}}=\frac{1}{t} \sum_{l=1}^{\infty} \frac{(-1)^{l}}{l^{k}}\left(1-e^{-t}\right)^{l}
$$




$$
\begin{gathered}
=\frac{1}{t} \sum_{l=1}^{\infty} \frac{(-1)^{l}}{[l]_{q}^{k}} l ! \sum_{p=l}^{\infty}(-1)^{p} S_{2}(p, l) \frac{t^{p}}{p !} \\
=\frac{1}{t} \sum_{p=1}^{\infty} \sum_{l=1}^{p} \frac{(-1)^{l+p}}{[l]_{q}^{k}} l ! S_{2}(p, l) \frac{t^{p}}{p !} \\
=\sum_{p=0}^{\infty}\left(\sum_{l=1}^{p+1} \frac{(-1)^{l+p+1}}{[l]_{q}^{k}} l ! \frac{S_{2}(p+1, l)}{p+1}\right) \frac{t^{p}}{p !} .
\end{gathered}
$$

From equations (2.7) and (2.8), we have

$$
\begin{gathered}
\sum_{n=0}^{\infty} H \beta_{n, q}^{(k)}(x, y ; \lambda) \frac{t^{n}}{n !} \\
=\sum_{p=0}^{\infty}\left(\sum_{l=1}^{p+1} \frac{(-1)^{l+p+1}}{[l]_{q}^{k}} l ! \frac{S_{2}(p+1, l)}{p+1}\right) \frac{t^{p}}{p !}\left(\sum_{n=0}^{\infty} H \beta_{n}(x, y ; \lambda) \frac{t^{n}}{n !}\right) .
\end{gathered}
$$

Replacing $n$ by $n-p$ in the r.h.s of above equation and comparing the coefficients of $t^{n}$, we get the result $(2.6)$.

Theorem 2.6. For $n \geq 1$, we have

$$
\begin{gathered}
H \beta_{n, q}^{(k)}(x+1, y ; \lambda)-{ }_{H} \beta_{n}^{(k)}(x, y ; \lambda) \\
=\sum_{p=1}^{n}\left(\begin{array}{c}
n \\
p
\end{array}\right)\left(\sum_{l=0}^{p-1} \frac{(-1)^{l+p+1}}{[l+1]_{q}^{k}}(l+1) ! S_{2}(p, l+1)\right) H_{n-p}(x, y ; \lambda) .
\end{gathered}
$$

Proof. Using the Definition (2.1), we have

$$
\begin{gathered}
\sum_{n=0}^{\infty} H \beta_{n, q}^{(k)}(x+1, y ; \lambda) \frac{t^{n}}{n !}-\sum_{n=0}^{\infty} H \beta_{n, q}^{(k)}(x, y ; \lambda) \frac{t^{n}}{n !} \\
=\frac{\operatorname{Li}_{k, q}\left(1-e^{-t}\right)}{(1+\lambda t)^{\frac{1}{\lambda}}-1}(1+\lambda t)^{\frac{x+1}{\lambda}}\left(1+\lambda t^{2}\right)^{\frac{y}{\lambda}}-\frac{\operatorname{Li}_{k, q}\left(1-e^{-t}\right)}{(1+\lambda t)^{\frac{1}{\lambda}}-1}(1+\lambda t)^{\frac{x}{\lambda}}\left(1+\lambda t^{2}\right)^{\frac{y}{\lambda}} \\
=\operatorname{Li}_{k, q}\left(1-e^{-t}\right)(1+\lambda t)^{\frac{x}{\lambda}}\left(1+\lambda t^{2}\right)^{\frac{y}{\lambda}} \\
=\sum_{l=0}^{\infty} \frac{\left(1-e^{-t}\right)^{l+1}}{[l+1]_{q}^{k}}(1+\lambda t)^{\frac{x}{\lambda}}\left(1+\lambda t^{2}\right)^{\frac{y}{\lambda}} \\
=\sum_{p=1}^{\infty}\left(\sum_{l=0}^{p-1} \frac{(-1)^{l+p+1}}{[l+1]_{q}^{k}}(l+1) ! S_{2}(p, l+1)\right) \frac{t^{p}}{p !}(1+\lambda t)^{\frac{x}{\lambda}}\left(1+\lambda t^{2}\right)^{\frac{y}{\lambda}} \\
=\left(\sum_{p=1}^{\infty}\left(\sum_{l=0}^{p-1} \frac{(-1)^{l+p+1}}{[l+1]_{q}^{k}}(l+1) ! S_{2}(p, l+1)\right) \frac{t^{p}}{p !}\right)\left(\sum_{n=0}^{\infty} H_{n}(x, y ; \lambda) \frac{t^{n}}{n !}\right) .
\end{gathered}
$$

Replacing $n$ by $n-p$ in the above equation and comparing the coefficients of $t^{n}$, we get the result (2.9). 
Theorem 2.7. For $n \geq 0, d \in \mathbb{N}$ and $k \in \mathbb{Z}$, we have

$$
\begin{aligned}
& { }_{H} \beta_{n, q}^{(k)}(x, y ; \lambda) \\
& =\sum_{a=0}^{d-1} \sum_{l=0}^{n} \sum_{p=1}^{l+1}\left(\begin{array}{c}
n \\
l
\end{array}\right) d^{n-l-1} \frac{(-1)^{l+p+1} p ! S_{2}(l+1, p)}{p^{k}[l+1]_{q}^{k}}{ }_{H} \beta_{n-l}\left(\frac{l+x}{d}, y ; \frac{\lambda}{d}\right) .
\end{aligned}
$$

Proof. From equation (2.1), we can be written as

$$
\begin{gathered}
\sum_{n=0}^{\infty}{ }_{H} \beta_{n, q}^{(k)}(x, y ; \lambda) \frac{t^{n}}{n !}=\frac{\operatorname{Li}_{k, q}\left(1-e^{-t}\right)}{(1+\lambda t)^{\frac{1}{\lambda}}-1}(1+\lambda t)^{\frac{x}{\lambda}}\left(1+\lambda t^{2}\right)^{\frac{y}{\lambda}} \\
=\frac{\operatorname{Li}_{k, q}\left(1-e^{-t}\right)}{(1+\lambda t)^{\frac{d}{\lambda}}-1} \sum_{a=0}^{d-1}(1+\lambda t)^{\frac{l+x}{\lambda}}\left(1+\lambda t^{2}\right)^{\frac{y}{\lambda}} \\
=\left(\frac{\operatorname{Li}_{k, q}\left(1-e^{-t}\right)}{t}\right) \frac{1}{d} \sum_{a=0}^{d-1} \frac{d t}{(1+\lambda t)^{\frac{d}{\lambda}}-1}(1+\lambda t)^{\frac{l+x}{\lambda}}\left(1+\lambda t^{2}\right)^{\frac{y}{\lambda}} \\
=\left(\sum_{l=0}^{\infty}\left(\sum_{p=1}^{l+1} \frac{(-1)^{l+p+1}}{p^{k}} p ! \frac{S_{2}(l+1, p)}{[l+1]_{q}^{k}}\right) \frac{t^{l}}{l !}\right)\left(\sum_{n=0}^{\infty} d^{n-1} \sum_{a=0}^{d-1} H \beta_{n}\left(\frac{l+x}{d}, y ; \frac{\lambda}{d}\right) \frac{t^{n}}{n !}\right) .
\end{gathered}
$$

Replacing $n$ by $n-l$ in above equation and comparing the coefficient of $t^{n}$, we get the result (2.10).

\section{Summation formulae for degenerate Hermite poly-Bernoulli polynomials with q-parameter}

For the derivation of implicit formulae involving degenerate q-polyBernoulli polynomials $\beta_{n, q}^{(k)}(x ; \lambda)$ and degenerate Hermite poly-Bernoulli polynomials ${ }_{H} \beta_{n, q}^{(k)}(x, y ; \lambda)$ the same considerations as developed for the ordinary Hermite and related polynomials in Khan [14] and Hermite-Bernoulli polynomials in Pathan and Khan [19], [20], [21], [22] holds as well. First we prove the following results involving degenerate Hermite poly-Bernoulli polynomials with q-parameter ${ }_{H} \beta_{n, q}^{(k)}(x, y ; \lambda)$.

Theorem 3.1. The following implicit summation formulae involving degenerate Hermite polynomials ${ }_{H} \beta_{n, q}^{(k)}(\lambda, \mu ; x, y)$ holds true:

$$
\begin{aligned}
& { }_{H} \beta_{m+n, q}^{(k)}(x, y ; \lambda) \\
& =\sum_{r, s=0}^{m, n}\left(\begin{array}{l}
m \\
r
\end{array}\right)\left(\begin{array}{l}
n \\
s
\end{array}\right)(x-v)^{r+s}\left[\frac{1}{\lambda} \log (1+\lambda)\right]^{r+s}{ }_{H} \beta_{m+n-r-s, q}^{(k)}(v, y ; \lambda) .
\end{aligned}
$$

Proof. Replacing $t$ by $t+u$ in (2.1) and rewrite the generating function (2.1) as

$$
\frac{\operatorname{Li}_{k, q}\left(1-e^{-(t+u)}\right)}{(1+\lambda(t+u))^{\frac{1}{\lambda}}-1} e^{\frac{y(t+u)^{2}}{\lambda}(\log (1+\lambda))}
$$




$$
=e^{-\frac{x(t+u)}{\lambda}(\log (1+\lambda)} \sum_{m, n=0}^{\infty} H \beta_{m+n, q}^{(k)}(x, y ; \lambda) \frac{t^{m}}{m !} \frac{t^{n}}{n !} .
$$

Upon replacing $x$ by $v$ in the above equation, it is not difficult to observe that

$$
\begin{gathered}
\sum_{m, n=0}^{\infty}{ }_{H} \beta_{m+n, q}^{(k)}(\lambda ; x, y) \frac{t^{m}}{m !} \frac{t^{n}}{n !}=e^{\frac{x(t+u)(x-v)}{\lambda} \log (1+\lambda)} \sum_{m, n=0}^{\infty}{ }_{H} \beta_{m+n, q}^{(k)}(\lambda ; v, y) \frac{t^{m}}{m !} \frac{t^{n}}{n !} \\
\sum_{m, n=0}^{\infty} H \beta_{m+n, q}^{(k)}(\lambda, \mu ; x, y) \frac{t^{p}}{p !} \frac{t^{q}}{q !} \\
=\sum_{N=0}^{\infty} \frac{\left[\frac{x(t+u)(x-v)}{\lambda} \log (1+\lambda)\right]^{N}}{N !} \sum_{p, q=0}^{\infty}{ }_{H} \beta_{p+q}^{(k)}(\lambda, \mu ; v, y) \frac{t^{p}}{p !} \frac{t^{q}}{q !} .
\end{gathered}
$$

Now, by applying the following known series identity [24, p. 52, Eq. 1.6(2)]:

$$
\begin{gathered}
\sum_{N=0}^{\infty} f(N) \frac{(x+y)^{N}}{N !}=\sum_{p, q=0}^{\infty} f(n+m) \frac{x^{p}}{p !} \frac{y^{q}}{q !} \\
\sum_{m, n=0}^{\infty} H \beta_{m+n, q}^{(k)}(\lambda ; x, y) \frac{t^{m}}{m !} \frac{t^{n}}{n !} \\
=\sum_{r, s=0}^{\infty}(x-v)^{r+s}\left[\frac{1}{\lambda} \log (1+\lambda)\right]^{r+s} \frac{t^{r}}{r !} \frac{u^{s}}{s !} \sum_{m, n=0}^{\infty}{ }_{H} \beta_{m+n, q}^{(k)}(\lambda ; v, y) \frac{t^{m}}{m !} \frac{t^{n}}{n !} .
\end{gathered}
$$

On replacing $m$ by $m-r$ and $n$ by $n-s$ in above equation, we get

$$
\begin{gathered}
\sum_{m, n=0}^{\infty} H \beta_{m+n, q}^{(k)}(\lambda ; x, y) \frac{t^{m}}{m !} \frac{t^{n}}{n !} \\
=\sum_{m, n=0}^{\infty} \sum_{r, s=0}^{p, q}(x-v)^{r+s}\left[\frac{1}{\lambda} \log (1+\lambda)\right]^{r+s} \beta_{m+n-r-s, q}^{(k)}(\lambda ; v, y) \frac{t^{m}}{(m-r) ! r !} \frac{t^{n}}{(n-s) ! s !} .
\end{gathered}
$$

Comparing the coefficients of $\frac{t^{m}}{m !}$ and $\frac{t^{n}}{n !}$, we get the result (3.1).

Theorem 3.2. For $x, y \in \mathbb{R}$ and $n \geq 0$. Then

$$
{ }_{H} \beta_{n, q}^{(k)}(x+u, y+w ; \lambda)=\sum_{m=0}^{n}\left(\begin{array}{c}
n \\
m
\end{array}\right){ }_{H} \beta_{n-m, q}^{(k)}(x, y ; \lambda) H_{m}(u, w ; \lambda) .
$$

Proof. By the definition of q-degenerate poly-Bernoulli polynomials and the definition (1.3), we have

$$
\begin{gathered}
\frac{\operatorname{Li}_{k, q}\left(1-e^{-t}\right)}{(1+\lambda t)^{\frac{1}{\lambda}}-1}(1+\lambda t)^{\frac{x+u}{\lambda}}\left(1+\lambda t^{2}\right)^{\frac{y+w}{\lambda}} \\
=\left(\sum_{n=0}^{\infty} H \beta_{n, q}^{(k)}(x, y ; \lambda) \frac{t^{n}}{n !}\right)\left(\sum_{m=0}^{\infty} H_{m}(u, w ; \lambda) \frac{t^{m}}{m !}\right) .
\end{gathered}
$$

Now replacing $n$ by $n-m$ and comparing the coefficients of $t^{n}$, we get the result (3.3). 
Theorem 3.3. For $x, y \in \mathbb{R}$ and $n \geq 0$. Then

${ }_{H} \beta_{n, q}^{(k)}(x, y ; \lambda)=\sum_{m=0}^{n-2 j} \sum_{j=0}^{\left[\frac{n}{2}\right]} \beta_{m, q}^{(k)}(\lambda)\left(-\frac{x}{\lambda}\right)_{n-m-2 j}(-\lambda)^{n-m-j}\left(-\frac{y}{\lambda}\right)_{j} \frac{n !}{m ! j !(n-2 j-m) !}$.

Proof. Applying the definition (2.1) to the term $\frac{\operatorname{Li}_{k, q}\left(1-e^{-t}\right)}{(1+\lambda t)^{\frac{1}{\lambda}}-1}$ and expanding the function $(1+\lambda t)^{\frac{x}{\lambda}}\left(1+\lambda t^{2}\right)^{\frac{y}{\lambda}}$ at $t=0$ yields

$$
\begin{gathered}
\frac{\operatorname{Li}_{k, q}\left(1-e^{-t}\right)}{(1+\lambda t)^{\frac{1}{\lambda}}-1}(1+\lambda t)^{\frac{x}{\lambda}}\left(1+\lambda t^{2}\right)^{\frac{y}{\lambda}} \\
=\left(\sum_{m=0}^{\infty} \beta_{m, q}^{(k)}(\lambda) \frac{t^{m}}{m !}\right)\left(\sum_{n=0}^{\infty}\left(-\frac{x}{\lambda}\right)_{n} \frac{(-\lambda t)^{n}}{n !}\right)\left(\sum_{j=0}^{\infty}\left(-\frac{y}{\lambda}\right)_{j} \frac{\left(-\lambda t^{2}\right)^{j}}{j !}\right) \\
=\sum_{n=0}^{\infty}\left(\sum_{m=0}^{n}\left(\begin{array}{c}
n \\
m
\end{array}\right) \beta_{m, q}^{(k)}(\lambda)\left(-\frac{x}{\lambda}\right)_{n-m}(-\lambda)^{n-m}\right) \frac{t^{n}}{n !}\left(\sum_{j=0}^{\infty}\left(-\frac{y}{\lambda}\right)_{j} \frac{\left(-\lambda t^{2}\right)^{j}}{j !}\right) .
\end{gathered}
$$

Replacing $n$ by $n-2 j$, we have

$$
\begin{gathered}
\sum_{n=0}^{\infty} H \beta_{n, q}^{(k)}(x, y ; \lambda) \frac{t^{n}}{n !} \\
=\sum_{n=0}^{\infty}\left(\sum_{m=0}^{n-2 j} \sum_{j=0}^{\left[\frac{n}{2}\right]}\left(\begin{array}{l}
n-2 j \\
m
\end{array}\right) \beta_{m, q}^{(k)}(\lambda)\left(-\frac{x}{\lambda}\right)_{n-m-2 j}(-\lambda)^{n-m-j}\left(-\frac{y}{\lambda}\right)_{j}\right) \frac{t^{n}}{(n-2 j) ! j !} .
\end{gathered}
$$

Equating their coefficients of $t^{n}$, we get the result (3.4).

Theorem 3.4. For $x, y \in \mathbb{R}$ and $n \geq 0$. Then

$$
{ }_{H} \beta_{n, q}^{(k)}(x, y ; \lambda)=\sum_{m=0}^{n}\left(\begin{array}{c}
n \\
m
\end{array}\right)\left(-\frac{z}{\lambda}\right)_{n-m}(-\lambda)^{n-m}{ }_{H} \beta_{m, q}^{(k)}(x-z, y ; \lambda) .
$$

Proof. By exploiting the generating function (2.1), we can write the equation

$$
\begin{gathered}
\sum_{n=0}^{\infty} H \beta_{n, q}^{(k)}(x, y ; \lambda) \frac{t^{n}}{n !}=\frac{\operatorname{Li}_{k, q}\left(1-e^{-t}\right)}{(1+\lambda t)^{\frac{1}{\lambda}-1}}(1+\lambda t)^{\frac{x-z}{\lambda}}\left(1+\lambda t^{2}\right)^{\frac{y}{\lambda}}(1+\lambda t)^{\frac{z}{\lambda}} . \\
=\left(\sum_{m=0}^{\infty} H \beta_{m, q}^{(k)}(x-z, y ; \lambda) \frac{t^{m}}{m !}\right)\left(\sum_{n=0}^{\infty}\left(-\frac{z}{\lambda}\right)_{n} \frac{(-\lambda t)^{n}}{n !}\right) .
\end{gathered}
$$

Replacing $n$ by $n-m$ in above equation and equating their coefficients of $t^{n}$ leads to formula (3.6). 
Theorem 3.5. The following implicit summation formula involving degenerate Hermite poly-Bernoulli polynomials with q-parameter ${ }_{H} \beta_{n, q}^{(k)}(x, y ; \lambda)$ holds true:

$$
{ }_{H} \beta_{n, q}^{(k)}(x+1, y ; \lambda)=\sum_{r=0}^{n}\left(\begin{array}{c}
n \\
r
\end{array}\right)\left(-\frac{1}{\lambda}\right)_{r}(-\lambda)^{r}{ }_{H} \beta_{n-r, q}^{(k)}(x, y ; \lambda) .
$$

Proof. By the definition of degenerate Hermite poly-Bernoulli polynomials with qparameter, we have

$$
\begin{gathered}
\sum_{n=0}^{\infty} H \beta_{n, q}^{(k)}(x+1, y ; \lambda) \frac{t^{n}}{n !}+\sum_{n=0}^{\infty}{ }_{H} \beta_{n, q}^{(k)}(x, y ; \lambda) \frac{t^{n}}{n !} \\
=\frac{\operatorname{Li}_{k, q}\left(1-e^{-t}\right)}{(1+\lambda t)^{\frac{1}{\lambda}}-1}(1+\lambda t)^{\frac{x}{\lambda}}\left(1+\lambda t^{2}\right)^{\frac{y}{\lambda}}\left((1+\lambda t)^{\frac{1}{\lambda}}+1\right) \\
=\left(\sum_{n=0}^{\infty} H \beta_{n, q}^{(k)}(x, y ; \lambda) \frac{t^{n}}{n !}\right)\left(\sum_{r=0}^{\infty}\left(-\frac{1}{\lambda}\right)_{r} \frac{(-\lambda t)^{r}}{r !}\right)+\sum_{n=0}^{\infty} H \beta_{n, q}^{(k)}(x, y ; \lambda) \frac{t^{n}}{n !} \\
=\sum_{n=0}^{\infty} \sum_{r=0}^{n} H \beta_{n-r, q}^{(k)}(x, y ; \lambda)\left(-\frac{1}{\lambda}\right)_{r}(-\lambda)^{r} \frac{t^{n}}{(n-r) ! r !}+\sum_{n=0}^{\infty} \beta_{n, q}^{(k)}(x, y ; \lambda) \frac{t^{n}}{n !} .
\end{gathered}
$$

Finally, equating the coefficients of the like powers of $t^{n}$, we get (3.8).

\section{General symmetry identities}

In this section, we establish symmetry identities for the q-degenerate polyBernoulli polynomials $\beta_{n, q}^{(k)}(x ; \lambda)$ and the degenerate Hermite poly-Bernoulli polynomials with q-parameter ${ }_{H} \beta_{n, q}^{(k)}(x, y ; \lambda)$ by applying the generating function(2.1) and (2.2). The results extend some known identities of Khan [13], [14], [15], [16], Pathan and Khan [19], [20], [21], [22].

Theorem 4.1. Let $a, b>0$ and $a \neq b$. For $x, y \in \mathbb{R}, n \geq 0,0 \leq q<1$, then the following identity holds true:

$$
\begin{aligned}
& \sum_{m=0}^{n}\left(\begin{array}{c}
n \\
m
\end{array}\right) b^{m} a_{H}^{n-m} \beta_{n-m, q}^{(k)}\left(b x, b^{2} y ; \lambda\right)_{H} \beta_{m, q}^{(k)}\left(a x, a^{2} y ; \lambda\right) \\
= & \sum_{m=0}^{n}\left(\begin{array}{c}
n \\
m
\end{array}\right) a^{m} b_{H}^{n-m} \beta_{n-m, q}^{(k)}\left(a x, a^{2} y ; \lambda\right)_{H} \beta_{m, q}^{(k)}\left(b x, b^{2} y ; \lambda\right) .
\end{aligned}
$$

Proof. Start with

$$
G(t)=\left(\frac{\operatorname{Li}_{k, q}\left(1-e^{-a t}\right) \operatorname{Li}_{k, q}\left(1-e^{-b t}\right)}{\left((1+\lambda t)^{\frac{a}{\lambda}}-1\right)\left((1+\lambda t)^{\frac{b}{\lambda}}-1\right)}\right)(1+\lambda t)^{\frac{a b x}{\lambda}}\left(1+\lambda t^{2}\right)^{\frac{a^{2} b^{2} y}{\lambda}} .
$$

Then the expression for $G(t)$ is symmetric in $a$ and $b$ and we can expand $G(t)$ into series in two ways to obtain

$$
G(t)=\sum_{n=0}^{\infty} H \beta_{n, q}^{(k)}\left(b x, b^{2} y ; \lambda\right) \frac{(a t)^{n}}{n !} \sum_{m=0}^{\infty} H_{H}^{(k)} \beta_{m, q}^{\left(a x, a^{2} y ; \lambda\right)} \frac{(b t)^{m}}{m !}
$$




$$
=\sum_{n=0}^{\infty}\left(\sum_{m=0}^{n}\left(\begin{array}{c}
n \\
m
\end{array}\right) a^{n-m} b_{H}^{m} \beta_{n-m, q}^{(k)}\left(b x, b^{2} y ; \lambda\right)_{H} \beta_{m, q}^{(k)}\left(a x, a^{2} y ; \lambda\right)\right) \frac{t^{n}}{n !} .
$$

On the similar lines we can show that

$$
\begin{gathered}
G(t)=\sum_{n=0}^{\infty}{ }_{H} \beta_{n, q}^{(k)}\left(a x, a^{2} y ; \lambda\right) \frac{(b t)^{n}}{n !} \sum_{m=0}^{\infty} H_{m, q}^{(k)}\left(b x, b^{2} y ; \lambda\right) \frac{(a t)^{m}}{m !} \\
=\sum_{n=0}^{\infty}\left(\sum_{m=0}^{n}\left(\begin{array}{c}
n \\
m
\end{array}\right) a^{m} b_{H}^{n-m} \beta_{n-m, q}^{(k)}\left(a x, a^{2} y ; \lambda\right)_{H} \beta_{m, q}^{(k)}\left(b x, b^{2} y ; \lambda\right)\right) \frac{t^{n}}{n !} .
\end{gathered}
$$

Comparing the coefficients of $\frac{t^{n}}{n !}$ on the right hand sides of the last two equations, we arrive at the desired result.

Remark 4.2. For $b=1$, Theorem 4.1 reduces to

$$
\begin{aligned}
& \sum_{m=0}^{n}\left(\begin{array}{c}
n \\
m
\end{array}\right) a_{H}^{n-m} \beta_{n-m, q}^{(k)}(x, y ; \lambda)_{H} \beta_{m, q}^{(k)}\left(a x, a^{2} y ; \lambda\right) \\
& =\sum_{m=0}^{n}\left(\begin{array}{c}
n \\
m
\end{array}\right) a_{H}^{m} \beta_{n-m, q}^{(k)}\left(a x, a^{2} y ; \lambda\right)_{H} \beta_{m, q}^{(k)}(x, y ; \lambda) .
\end{aligned}
$$

Theorem 4.3. For all integers $a>0, b>0$ and $n \geq 0,0 \leq q<1$, the following identity holds true:

$$
\begin{aligned}
& \sum_{m=0}^{n}\left(\begin{array}{l}
n \\
m
\end{array}\right) a^{n-m} b_{H}^{m} \beta_{n-m, q}^{(k)}\left(b x, b^{2} z ; \lambda\right) \sum_{i=0}^{m}\left(\begin{array}{l}
m \\
i
\end{array}\right) \sigma_{i}(a-1 ; \lambda) \beta_{m-i, q}^{(k)}(a y ; \lambda) \\
= & \sum_{m=0}^{n}\left(\begin{array}{l}
n \\
m
\end{array}\right) a^{m} b_{H}^{n-m} \beta_{n-m, q}^{(k)}\left(a x, a^{2} z ; \lambda\right) \sum_{i=0}^{m}\left(\begin{array}{l}
m \\
i
\end{array}\right) \sigma_{i}(b-1 ; \lambda) \beta_{m-i, q}^{(k)}(b y ; \lambda),
\end{aligned}
$$

where generalized falling factorial sum $\sigma_{k}(n ; \lambda)$ is given by (1.15).

Proof. We now use

$$
H(t)=\frac{\operatorname{Li}_{k, q}\left(1-e^{-a t}\right) \operatorname{Li}_{k, q}\left(1-e^{-b t}\right)\left((1+\lambda t)^{\frac{a b}{\lambda}}-1\right)(1+\lambda t)^{\frac{a b(x+y)}{\lambda}}\left(1+\lambda t^{2}\right)^{\frac{a^{2} b^{2} z}{\lambda}}}{\left((1+\lambda t)^{\frac{a}{\lambda}}-1\right)\left((1+\lambda t)^{\frac{b}{\lambda}}-1\right)^{2}}
$$

to find that

$$
\begin{gathered}
g(t)=\left(\frac{\operatorname{Li}_{k, q}\left(1-e^{-a t}\right)}{(1+\lambda t)^{\frac{a}{\lambda}}-1}\right)(1+\lambda t)^{\frac{a b x}{\lambda}}\left(1+\lambda t^{2}\right)^{\frac{a^{2} b^{2} z}{\lambda}}\left(\frac{(1+\lambda t)^{\frac{a b}{\lambda}}-1}{(1+\lambda t)^{\frac{b}{\lambda}}-1}\right) \\
\times\left(\frac{\operatorname{Li}_{k, q}\left(1-e^{-b t}\right)}{(1+\lambda t)^{\frac{b}{\lambda}}-1}\right)(1+\lambda t)^{\frac{a b y}{\lambda}} \\
=\sum_{n=0}^{\infty} H \beta_{n, q}^{(k)}\left(b x, b^{2} z ; \lambda\right) \frac{(a t)^{n}}{n !} \sum_{n=0}^{\infty} \sigma_{n}(a-1 ; \lambda) \frac{(b t)^{n}}{n !} \sum_{n=0}^{\infty} \beta_{n, q}^{(k)}(a y ; \lambda) \frac{(b t)^{n}}{n !} .
\end{gathered}
$$


Using a similar plan, we get

$$
g(t)=\sum_{n=0}^{\infty} H \beta_{n, q}^{(k)}\left(a x, a^{2} z ; \lambda\right) \frac{(b t)^{n}}{n !} \sum_{n=0}^{\infty} \sigma_{n}(b-1 ; \lambda) \frac{(a t)^{n}}{n !} \sum_{n=0}^{\infty} \beta_{n, q}^{(k)}(b y ; \lambda) \frac{(a t)^{n}}{n !} .
$$

By comparing the coefficients of $t^{n}$ on the right hand sides of the last two equations, we arrive at the desired result.

\section{Conclusion}

The degenerate Hermite-poly-Bernoulli polynomials with q-parameter ${ }_{H} \beta_{n, q}^{(k)}(x, y ; \lambda)$ plays a major role in obtaining new expansions, identities and representations. We can introduce and study a class of related generalized polynomials by defining degenerate Gould-Hopper-poly-Bernoulli polynomials with q-parameter

$$
\frac{\operatorname{Li}_{k, q}\left(1-e^{-t}\right)}{(1+\lambda t)^{\frac{1}{\lambda}}-1}(1+\lambda t)^{\frac{x}{\lambda}}\left(1+\lambda t^{r}\right)^{\frac{y}{\lambda}}=\sum_{n=0}^{\infty} H \beta_{n, q}^{(k, r)}(x, y ; \lambda) \frac{t^{n}}{n !} .
$$

The equation (2.1) may be derived from (5.1) for $r=2$.

This process can easily be extended to establish degenerate multi-variable Hermite-poly-Bernoulli polynomials with q-parameter and Apostle type Bernoulli polynomials.

\section{References}

[1] Andrews, L.C., Special functions for engineers and mathematicians, Macmillan Co. New York, 1985.

[2] Bayad, A., Hamahata, Y., Multiple-polylogarithms and multi-poly-Bernoulli polynomials, Funct. Approx. Comment. Math., 46(2012), 45-61.

[3] Bell, E.T., Exponential polynomials, Ann. of Math., 35(1934), 258-277.

[4] Carlitz, L., Degenerate Stirling Bernoulli and Eulerian numbers, Util. Math., 15(1979), 51-88.

[5] Cenkcia, M., Komatsu, T., Poly-Bernoulli numbers and polynomials with a q-parameter, Journal of Number Theory, 152(2015), 38-54.

[6] Cesarano, C., Cennamo, G.M., Placidi, L., Humbert polynomials and functions in terms of Hermite polynomials towards applications to wave propagation, WSEAS Transactions on Mathematics, 14(2014), 595-602.

[7] Dattoli, G., Lorenzutta, S., Ricci, P.E., Cesarano, C., On a family of hybrid polynomials, Integral Trasforms and Special Functions, 15(2004), 485-490.

[8] Dattoli, G., Lorenzutta, S., Cesarano, C., Finite sums and generalized forms of Bernoulli polynomials, Rendiconti di Mathematica, 19(1999), 385-391.

[9] Dattoli, G., Srivastava, H.M., Cesarano, C., The Laguerre and Legendre polynomials from an operational point of view, Applied Mathematics and Computation, 124(2001), $117-127$. 
[10] Jolany, H., Mohebbi, H., Some results on generalized multi-poly Bernoulli and Euler polynomials, Int. J. Math. Comb., 2(2011), 117-129.

[11] Jung, N.S., Ryoo, C.S., Symmetric identities for degenerate q-poly-Bernoulli numbers and polynomials, J. Appl. Math. \& Informatics, 36(2018), 29-38.

[12] Kaneko, M., Poly-Bernoulli numbers, J. de Theorie de Nombres, 9(1997), 221-228.

[13] Khan, W.A., A note on degenerate Hermite-poly numbers and polynomials, J. Class. Anal., 1(2016), 65-76.

[14] Khan, W.A., Some properties of the generalized Apostol type Hermite-Based polynomials, Kyungpook Math. J., 55(2015), 597-614.

[15] Khan, W.A., A note on Hermite-based poly-Euler and multi poly-Euler polynomials, Palestine J. Math., 5(2016), 17-26.

[16] Khan, W.A., A new class of Hermite poly-Genocchi polynomials, J. Anal. and Number Theory, 4(2016), 1-8.

[17] Kim, D.S., Dolgy, T., Komatsu, D.V., Barnes type degenerate Bernoulli polynomials, Adv. Stud. Contemp. Math., 25(2015), 121-146.

[18] Kim, D.S., Kim, T., A note on degenerate poly-Bernoulli numbers and polynomials, Advanc. Diff. Equat., (2015), DOI 10.1186/s13662-015-0595-3.

[19] Pathan, M.A., Khan, W.A., Some implicit summation formulas and symmetric identities for the generalized Hermite-Bernoulli polynomials, Mediterr. J. Math., 12(2015), 679695.

[20] Pathan, M.A., Khan, W.A., A new class of generalized polynomials associated with Hermite and Euler polynomials, Mediterr. J. Math., 13(2016), 913-928.

[21] Pathan, M.A., Khan, W.A., Some implicit summation formulas and symmetric identities for the generalized Hermite-Euler polynomials, East-West J. Maths., 16(2014), 92-109.

[22] Pathan, M.A., Khan, W.A., Some new classes of generalized Hermite-based ApostolEuler and Apostol-Genocchi polynomials, Fasciculli Math., 55(2015), 153-170.

[23] Ryoo, C.S., On degenerate q-tangent polynomials of higher order, J. Appl. Math. \& Informatics, 35(2017), 113-120.

[24] Srivastava, H.M., Manocha, H.L., A Treatise on Generating Functions, Ellis Horwood Limited, New York, 1984.

[25] Young, P.T., Degenerate Bernoulli polynomials generalized factorials sums and their application, J. Number Theory, 128(2008), 738-758.

Waseem A. Khan

Department of Mathematics and Natural Sciences

Prince Mohammad Bin Fahd University,

P.O. Box 1664, Al Khobar 31952, Saudi Arabia

e-mail: wkhan1@pmu.edu.sa

Idrees A. Khan

Department of Mathematics, Faculty of Science, Integral University

Lucknow-226026, India

e-mail: khanidrees077@gmail.com

Musharraf Ali

Department of Mathematics, G.F. College, Shahjahanpur-242001, India

e-mail: drmusharrafali@gmail.com 